\title{
An electron-based crystalline undulator
}

\author{
M Tabrizi ${ }^{1}$, A V Korol ${ }^{1,2}$, A V Solov'yov ${ }^{1}$ and Walter Greiner ${ }^{1}$ \\ ${ }^{1}$ Frankfurt Institute for Advanced Studies, Johann Wolfgang Göthe-Universität, \\ Max-von-Laue-Str. 1, 60438 Frankfurt am Main, Germany \\ 2 Department of Physics, St. Petersburg State Maritime Technical University, \\ Leninskii prospect 101, St. Petersburg 198262, Russia \\ E-mail: tabrizi@bk.ru, a.korol@fias.uni-frankfurt.de, \\ solovyov@fias.uni-frankfurt.de
}

\begin{abstract}
We discuss the features of a crystalline undulator of the novel type based on the effect of a planar channeling of ultra-relativistic electrons in a periodically bent crystals. It is demonstrated that an electron-based undulator is feasible in the tens of $\mathrm{GeV}$ range of the beam energies, which is noticeably higher than the energy interval allowed in a positron-based undulator. Numerical analysis of the main parameters of the undulator as well as the characteristics of the emitted undulator radiation is carried out for 20 and $50 \mathrm{GeV}$ electrons channeling in diamond and silicon crystals along the (111) crystallographic planes.

PACS numbers: 41.60.-m, 61.82.Rx, 61.85.+p
\end{abstract}




\section{Introduction}

In this paper we suggest and discuss a new type of a powerful source of high energy photons generated by a bunch of ultra-relativistic electrons undergoing planar channeling in a periodically bent crystal. We call such system 'an electron-based crystalline' undulator. The feasibility of the undulator has been recently proven for the first time in [1]. In this work the results of more detailed qualitative and quantitative analysis of the radiation formed in electron-based crystalline undulators at different energies of electron and for different types of crystals are presented.

As it is known [2,3], in a crystalline undulator there appears, in addition to the wellknown channeling radiation [4], the radiation of an undulator type which is due to the periodic motion of channeling particles which follow the bending of the crystallographic planes. In the cited papers, as well as in the subsequent publications [5-8], a feasibility to create a short-wave crystalline undulator emitting intensive and monochromatic radiation, based on an ultra-relativistic positron channeling was proven (for the latest review see [8]). More recently [9] it was shown that the brilliance of the radiation from a positron-based crystalline undulator in the energy range from hundreds of keV up to tens of $\mathrm{MeV}$ is comparable to that of a conventional light source of the third generation but for much lower photon energies. Experimental study of this phenomenon is on the way within the framework of the PECU project [10].
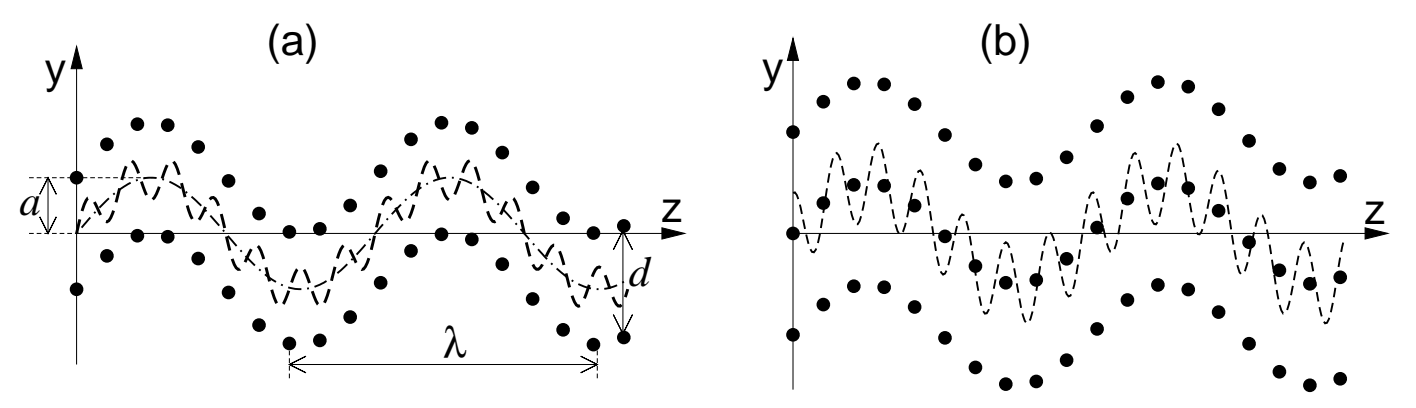

Figure 1. Schematic representation of the positron-based (graph (a)) and the electron-based (graph (b)) crystalline undulators. Circles denote the atoms belonging to neighbouring crystallographic planes (separated by the distance $d$ ) which are periodically bent. The wavy lines show the trajectories of channeling particles. A positron channels between two neighbouring planes, whereas the electron channeling occurs nearby the crystallographic plane. The profile of periodic bending is given by $y(z)=a \sin (2 \pi z / \lambda)$, where $\lambda$ and $a$ are the period and amplitude of the bending (indicated in graph (a)).

The operational principle of a crystalline undulator does not depend on the type of a projectile and is illustrated by figure 1. Under certain conditions $[2,3]$ an ultrarelativistic charged particle, which enters the crystal at the angle smaller than the Lindhard's critical angle [11], will penetrate through the crystal following the bendings of its planes. Consequently, the trajectory of the particle contains two elements. Firstly, there are oscillations due to the action of the interplanar force, - the channeling 
oscillations [11], whose frequency $\Omega_{\mathrm{ch}}=c \sqrt{2 U_{\max }^{\prime} / d \varepsilon}$ ( $c$ is the speed of light) depends on the energy of the particle $\varepsilon$ and the parameters of the channel: the maximal gradient of the interplanar potential $U_{\max }^{\prime}$ and the interplanar distance $d$. Secondly, there are oscillations due to the periodicity of the bendings, the undulator oscillations, whose frequency is $\omega_{0} \approx 2 \pi c / \lambda$, where $\lambda$ is the spatial period of bending. The spontaneous emission is associated with both of these oscillations. Typical frequencies of the channeling and undulator radiation are $\omega_{\text {ch }} \approx 2 \gamma^{2} \Omega_{\text {ch }}$ and $\omega \approx 4 \gamma^{2} \omega_{0} /\left(2+p^{2}\right)$, where $\gamma=\varepsilon / m c^{2}$ is the Lorentz factor of the particle and $p=2 \pi \gamma a / \lambda$ is the undulator parameter, $a$ is the amplitude of bending. If $\omega_{0} \ll \Omega_{\mathrm{ch}}$, then the frequencies of channeling and undulator radiation are well separated. In this case the characteristics of undulator radiation are practically independent on channeling oscillations [2,3], and the operational principle of a crystalline undulator is the same as for a conventional one [12-14], in which the monochromaticity of the radiation is the result of a constructive interference of the photons emitted from similar parts of trajectory.

It was shown in $[2,3]$ that a crystalline undulator can operate provided the following conditions are met:

$$
\begin{cases}C=4 \pi^{2} \varepsilon a / U_{\max }^{\prime} \lambda^{2}<1 & \text { - stable channeling, } \\ d<a \ll \lambda & \text { - large-amplitude regime, } \\ N=L / \lambda \gg 1 & \text { - large number of periods, } \\ L \sim \min \left[L_{\mathrm{d}}(C), L_{\mathrm{a}}(\omega)\right] & \text { - account for dechanneling and photon attenuation, } \\ \Delta \varepsilon / \varepsilon \ll 1 & \text { - low radiative losses. }\end{cases}
$$

Earlier, it was demonstrated that these conditions can be achieved in a positron-based undulator $[2,3,8]$. In the present work, we demonstrate this for an electron-based one. Prior to doing this let us present a short description of the physics lying behind these conditions.

A stable channeling of a projectile in a periodically bent channel occurs if the maximal centrifugal force in the channel $F_{\text {cf }}$ is less than the maximal interplanar force $U_{\max }^{\prime}$, i.e. $C \equiv F_{\mathrm{cf}} / U_{\max }^{\prime}<1$. For an ultra-relativistic particle $F_{\mathrm{cf}} \approx \varepsilon / R_{\min }$, where $R_{\text {min }}=\lambda^{2} / 4 \pi^{2} a$ is the minimum curvature radius of the channel with the profile of the periodical bendings given by $y(z)=a \sin (2 \pi z / \lambda)$ (see figure 1).

The operation of a crystalline undulator must be considered in the large-amplitude regime. Omitting the discussion (see $[2,3,8]$ ) we note, that the limit $a / d>1$ accompanied by the condition $C \ll 1$ is mostly advantageous, since in this case the typical frequencies of undulator and channeling radiation are well separated, and the latter does not affect the parameters of the former, whereas the intensity of the undulator radiation becomes comparable or higher than that of the channeling radiation. On the other hand, the inequality $a \ll \lambda$ means that the crystal structure is not destroyed and the deformation is an elastic one. Also, this results in moderate values of the undulator parameter, $p \sim 1$, which ensure that the emitted radiation is of the undulator type rather than of the synchrotron one. Consequently, the quantities $a, d$ and $\lambda$ must satisfy the 
double inequality presented in the second line in (1).

The term 'undulator' signifies that the number of periods is large, $N \gg 1$. This inequality leads to a very peculiar pattern of a spectral-angular distribution of the emitted radiation. Namely, for each value of the emission angle $\theta$ (measured with respect to the undulator axis) the spectrum consists of a set of narrow, well-separated peaks (harmonics). In a conventional undulator, based on the action of a magnetic (or electric) field [12-14], the beams of charged particles and photons move in vacuum. In such 'ideal' conditions the peak intensity of the emitted radiation is proportional to $N^{2}$.

In a crystalline undulator the beams move in a medium and are affected by the dechanneling and photon attenuation effects, which noticeably reduce the $N^{2}$ increase of the peak intensity [7]. Due to collisions with the crystal constituents the channeling particle increases its transverse energy $\varepsilon_{\perp}$. At some point $\varepsilon_{\perp}$ exceeds the interplanar potential barrier and leaves the channel. The average interval for a particle to penetrate into a crystal until it dechannels is called the dechanneling length $L_{\mathrm{d}}$. In a straight crystal $L_{\mathrm{d}}$ depends on its type, the energy and the type of a projectile. In addition to these, in a periodically bent crystal $L_{\mathrm{d}}$ acquires the dependence on the parameter $C$ [3]. The dechanneling length introduces a natural upper limit on the length of a crystalline undulator: $L \leq L_{\mathrm{d}}(C)$. The essential difference between an electron-based undulator and a positron-based one lies in the fact that the dechanneling process for these projectiles occurs differently, resulting in a strong inequality $L_{\mathrm{d}}^{\mathrm{e}^{-}} \ll L_{\mathrm{d}}^{\mathrm{e}^{+}}[15]$. We discuss this important issue in more detail in section 2 .

The photon attenuation stands for a decrease of the intensity of the emitted radiation in the crystal due to the processes of absorption and scattering. The interval within which the intensity decreases by a factor of $e$ is called the attenuation length, $L_{\mathrm{a}}(\omega)$ [16]. This quantity is tabulated for a number of elements and over wide ranges of photon frequencies [17]. It is worth noting that for sufficiently high photon energies $\left(\hbar \omega \geq 10^{2} \mathrm{keV}\right.$ ) the restriction on the length of undulator due to the photon attenuation effect becomes much less severe than to the dechanneling process $[2,3,8]$.

It was demonstrated $[7,9]$ that in the limit $L \gg L_{\mathrm{d}}(C)$ the intensity of radiation is defined not by the total number of undulator periods $N=L / \lambda$, but rather by the quantity $N_{\mathrm{d}}=L_{\mathrm{d}}(C) / \lambda$ which is the number of periods within the dechanneling length. Since for an ultra-relativistic particle $L_{\mathrm{d}} \propto \varepsilon$ [18-20], it seems natural that to increase $N_{\mathrm{d}}$ one can consider higher energies. However, at higher energies another limitation appears $[2,3,6]$. The coherence of undulator radiation is only possible when the energy loss $\Delta \varepsilon$ of the particle during its passage through the undulator is small, $\Delta \varepsilon \ll \varepsilon$. This statement together with the fact, that for an ultra-relativistic projectile $\Delta \varepsilon$ is mainly due to radiation [18], leads to the conclusion that $L$ must be much smaller than the radiation length $L_{\mathrm{r}}$, which defines the mean energy loss of an ultra-relativistic particle per unit length (see e.g. [18]). Therefore, we come to the forth and fifth limitations in (11).

A thorough analysis of the conditions (11) for the system "periodically bent crystal + ultra-relativistic positrons" was performed for the first time in [2,3] (and analyzed 
further in $[5,6,8,9])$. The ranges of $\varepsilon, a$ and $\lambda$ for a number of crystals were established within which the operation of the crystalline undulator is possible. These ranges include $\varepsilon=(0.5 \ldots 5) \mathrm{GeV}, a / d=10^{1} \ldots 10^{2}, C=0.01 \ldots 0.2, N \sim N_{\mathrm{d}}=10^{1} \ldots 10^{2}$, $\omega \geq 10^{2} \mathrm{keV}$ and are common for all investigated crystals. The importance of the above mentioned regime in application to a positron-based undulator was later realized by other authors $[21,22]$.

In the case of electron channeling the restriction due to the dechanneling effect on the parameters of undulator are much more severe $[2,3,8]$. Therefore it has been commonly acknowledged that the concept of an electron-based undulator cannot be realized. In what follows we demonstrate that the crystalline undulator of this type is feasible but operates in the regime of higher beam energies than the positron-based undulator.

\section{Dechanneling length of positrons and electrons in a periodically bent crystal}

As mentioned, random multiple scattering of a channeling particle by electrons and nuclei of the crystal leads to the dechanneling effect. As a result, the volume density $n(z)$ of the channeling particles decreases with the penetration distance $z$. The dependence $n(z)$ can be approximated as $n(z)=n_{0} \exp \left(-z / L_{\mathrm{d}}\right)[3,4,19]$, where $n_{0}$ is the density at the entrance point. It follows from here that the dechanneling length $L_{\mathrm{d}}(C)$ is a natural limitation for the effective length of a crystalline undulator [3,7]. Therefore, the crystalline undulator is feasible when $L_{\mathrm{d}}(C)$ is large enough to ensure that the number of undulator periods within the dechanneling length satisfies the condition $N_{\mathrm{d}}=L_{\mathrm{d}}(C) / \lambda \gg 1$.

It is known that the dechanneling length of positrons exceeds that of electrons (see, e.g., [15]). This is due to the difference in the channeling processes for these projectiles. Indeed, positrons, possessing positive charge, are repulsed by crystal atoms and, thus, channel between neighbouring crystallographic planes (see figure 1(a)), where the concentration of nuclei and electrons is low. In contrast, electrons channel in close vicinity of atomic planes (figure 1(b)) where the number of the collisions with the crystal constituents is much larger. Therefore, electrons dechannel faster.

Let us discuss the approximations which one can use to calculate the dechanneling lengths of a positron and an electron.

The influence of the dechanneling process on the photon emission in a positronbased crystalline undulator was considered in $[2,3,7]$. In the cited works the expression for the dechanneling length of ultra-relativistic positrons in a periodically bent crystal, based of the diffusion model [19], was written as follows:

$$
L_{\mathrm{d}}(C)=(1-C)^{2} L_{\mathrm{d}}(0),
$$

where $L_{\mathrm{d}}(0)$ is the dechanneling length a straight channel. This quantity can be estimated as $L_{\mathrm{d}}(0)=\left(256 / 9 \pi^{2}\right)\left(a_{\mathrm{TF}} d \gamma / r_{0} \Lambda_{\mathrm{c}}\right)[3,19]$, where $r_{0}$ and $a_{\mathrm{TF}}$ are the 
classical radius of the electron and the Thomas-Fermi radius of the crystal atom, and $\Lambda_{\mathrm{c}}=\ln \left(\sqrt{2 \gamma} m c^{2} / I\right)-23 / 24$ ( $I$ stands for an average ionization potential of the atom).

The factor $(1-C)^{2}$ takes into account the decrease of the interplanar potential well due to periodic bendings. The advantage of the approximate formula (2) is that it explicitly demonstrates the dependence of the dechanneling length on $C, \varepsilon$ and parameters of the channel. Equation (2) was tested in [7] against more rigorous calculations, based on the simulation procedure of the positron channeling in straight and bent crystals. It was demonstrated that in wide range of the parameters (2) produces a good quantitative estimate for $L_{\mathrm{d}}(C)$.

To estimate the dechanneling length for electrons, one can use the model due to Baier and Katkov [18] which relates $L_{\mathrm{d}}(0)$ to a mean square of the multiple scattering angle of an ultra-relativistic electron. Since electrons channel in close vicinity of atomic planes, the multiple scattering occur predominantly from the nuclei of the crystal. The latter makes the main contribution to the increase of $\varepsilon_{\perp}[18]$. Let $q$ denote the mean square of the multiple scattering angle per a unit length. Then, $L_{\mathrm{d}}(0)$ can be defined as the length within which the mean square of the multiple scattering angle becomes equal to the square of Lindhard angle $\theta_{\mathrm{L}}$, i.e.

$$
L_{\mathrm{d}}(0)=\theta_{\mathrm{L}}^{2} / q \text {. }
$$

Taking into account that individual scattering events are independent and using the small-angle scattering approximation at high energies [23], one estimates $q$ as follows [18]

$$
q \simeq \frac{2 \pi m^{2} c^{4}}{\alpha \varepsilon^{2}} L_{\mathrm{r}}^{-1}
$$

Here $\alpha$ is the fine structure constant, $L_{\mathrm{r}}$ is the radiation length in an amorphous medium. Using (44) in (3) and recalling that $\theta_{\mathrm{L}}=\sqrt{2 \Delta U / \varepsilon}(\Delta U$ stands for the depth of the interplanar potential well), one derives the following expression for the dechanneling length of an ultra-relativistic electron in a straight crystal [18]

$$
L_{\mathrm{d}}(0) \simeq \frac{\alpha}{\pi} \frac{\Delta U \varepsilon}{m^{2} c^{4}} L_{\mathrm{r}}
$$

This equation has been derived assuming that the dechanneling length of ultrarelativistic electrons in a straight crystal is less than the characteristic length of the radiation losses [18].

As far as we know (see also [24]), experimental measurements of the dechanneling length during planar channeling of high energy electrons were performed in [25-27]. In $[25,26]$ the dechanneling length for $1.2 \mathrm{GeV}$ electrons, channeled in $\mathrm{Si}(110)$ was determined as $L_{\mathrm{d}}(0)=25 \pm 5 \mu \mathrm{m}$. This value, as indicated in [25], is in a good agreement with (5). However, in the experiment [27] the dechanneling length for $350 \mathrm{MeV}$ electrons channeled in $\mathrm{Si}(110)$ was found as $L_{\mathrm{d}}(0)=31 \mu \mathrm{m}$, which is even greater than the value based on (5). Therefore, we believe that the approximate formula (5) can be used for a quantitative estimate of the dechanneling length of ultra-relativistic electrons in straight crystals. 
Figure 2 presents the dependence of $L_{\mathrm{d}}(0)$ on $\varepsilon$ for planar channeling of electrons, calculated in accordance with (5), and of positrons (see (2) for $C=0$ ) in various straight channels. Horizontal lines show the radiation length in the crystals. To calculate $L_{\mathrm{r}}$ we used equation (27.22) from [16]. It is seen from figure 2 that for all energies the dechanneling length for a positron exceeds that for an electron by more than an order of magnitude: $L_{\mathrm{d}}^{\mathrm{e}^{+}} / L_{\mathrm{d}}^{\mathrm{e}^{-}} \sim 10^{1} \ldots 10^{2}$. A positron-based crystalline undulator can be considered for $\varepsilon \leq 10 \mathrm{GeV}$, where the radiation length greatly exceeds $L_{\mathrm{d}}$. One can demonstrate that in this case it is possible to achieve $N_{\mathrm{d}} \sim 10^{1} \ldots 10^{2}[8,9]$. The corresponding values of the undulator period are $\lambda=L_{\mathrm{d}} / N_{\mathrm{d}}=10^{-4} \ldots 10^{-2} \mathrm{~cm}$. From figure 2 it is seen that this is exactly the interval to which $L_{\mathrm{d}}^{\mathrm{e}^{-}}$belongs. Therefore, for an electron of the energy much smaller than $10 \mathrm{GeV}$ the number of undulator periods within the dechanneling length is equal, in the order of magnitude, to one. Hence, an electron-based crystalline undulator can hardly be realized in this energy regime.

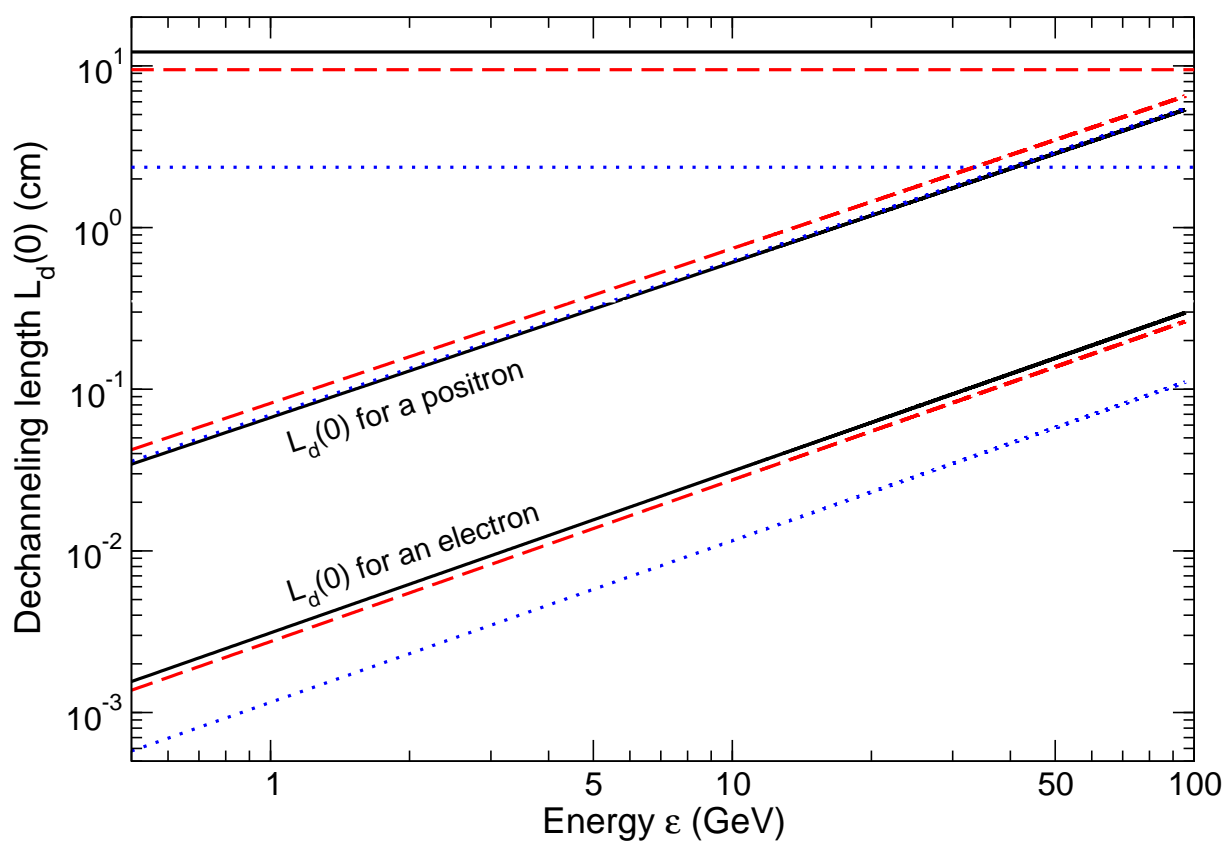

Figure 2. Dependence of the dechanneling length of ultra-relativistic electrons and positrons on energy in straight (111) planar channels of C (solid line), Si (dashed line), $\mathrm{Ge}($ dotted line). Horizontal lines denote the radiation lengths in the corresponding crystals.

For higher energies, $\varepsilon>10 \mathrm{GeV}$, the dechanneling length of a positron becomes comparable with the radiation length. This means, that a positron-based crystalline undulator cannot be realized due to large radiation losses (see the last condition in (1)). On the other hand, the dechanneling length of electrons at such energies, being much lower than $L_{\mathrm{r}}$, becomes comparable with $L_{\mathrm{d}}$ for positrons but of lower energies. Therefore, an electron-based crystalline undulator is meaningful to discuss within the interval $\varepsilon \sim 10^{1} \ldots 10^{2} \mathrm{GeV}$.

To conclude this section let us discuss a model which allows one to define the 
dechanneling length of an electron in a periodically bent crystal.

The factor $(1-C)^{2}$ in (2) appears as a result of application of the harmonic approximation for interplanar potential $U(x)[6,8,19]$. This approximation is adequate for a positively charged projectile, but its validity is not obvious for an electron. In the latter case the interplanar potential is strongly anharmonic [15]. To calculate the dependence of dechanneling length on $C$ one can consider the following arguments. In the point of maximum curvature, the effective potential, acting on the electron, can be written as $U_{C}(x)=U(x)-C U_{\max }^{\prime} x$, where $x$ is the distance from the plane. The depth of the effective potential well, $\Delta U_{C}$, defines the maximum value of the transverse energy which an electron may gain. Within the framework of the diffusion theory [19] the dechanneling length $L_{\mathrm{d}}(C)$ of an ultra-relativistic projectile in a bent channel is proportional to $\Delta U_{\mathrm{C}}$. Hence one can write $L_{\mathrm{d}}(C)=k(C) L_{\mathrm{d}}(0)$, where $L_{\mathrm{d}}(0)$ is defined by equation (5) and $k(C)$ stands for the ratio $\Delta U_{\mathrm{C}} / \Delta U<1$. To obtain the explicit dependence $k(C)$ one has to calculate the quantity $\Delta U_{\mathrm{C}}$ using a realistic model for $U(x)$.

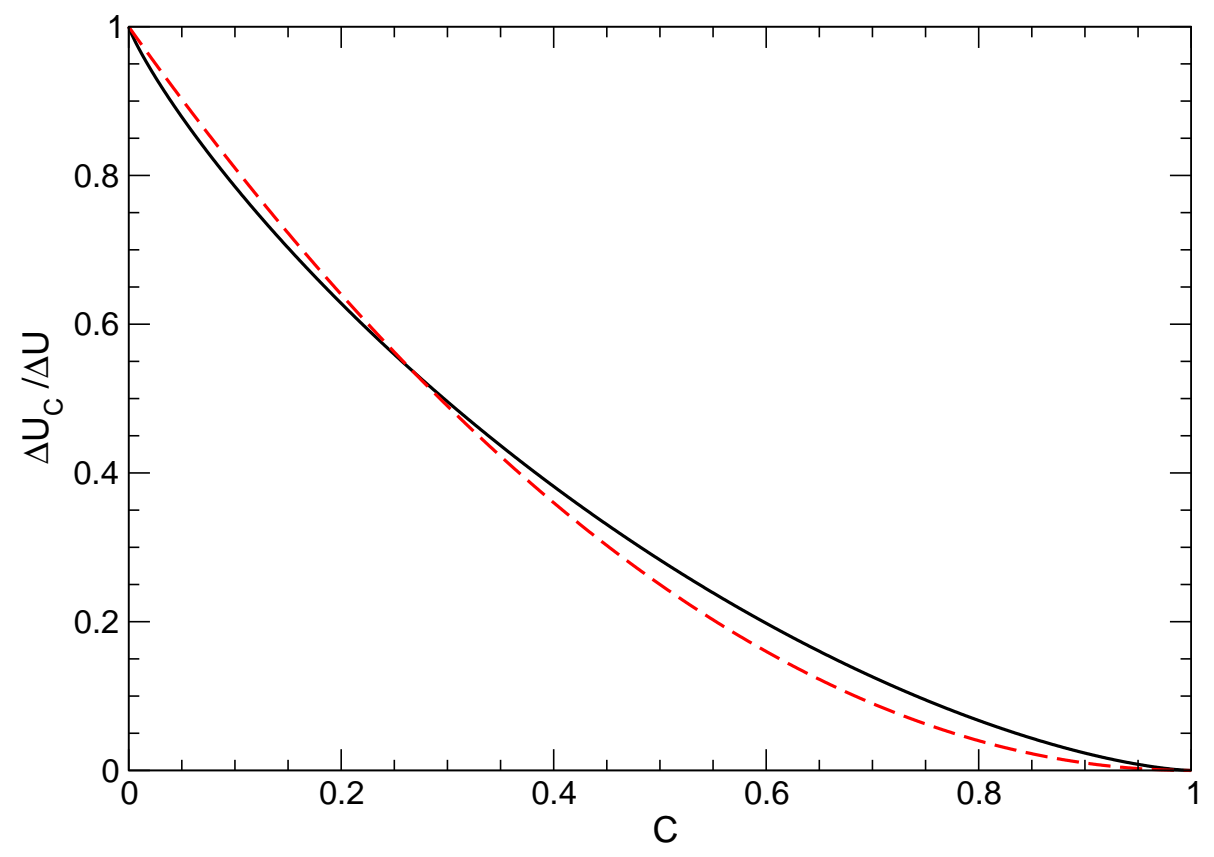

Figure 3. $\Delta U_{C} / \Delta U$ versus $C$ for the Pöschl-Teller potential (solid curve) and $(1-C)^{2}$ (dashed curve).

In our estimates we used the Pöschl-Teller potential [18]. The results of calculation are presented in figure 3, where the solid curve stands for $k(C)$ within the PöschlTeller model. The dashed curve presents the dependence $(1-C)^{2}$. It is seen that both curves are close for all $C$. Therefore, to estimate the dechanneling length of an ultra-relativistic electron in a periodically bent crystal one can use equation (2) with $L_{\mathrm{d}}(0)$ from (5). Consequently, the condition of a large number of periods within the dechanneling acquires the form

$$
N_{\mathrm{d}}=(1-C)^{2} L_{\mathrm{d}}(0) / \lambda \gg 1 \text {. }
$$


In the next section we use equations (2), (5) and (6) to calculate the characteristics of an electron-based crystalline undulator.

\section{Numerical results for the electron-based crystalline undulator}

To prove the feasibility of an electron-based crystalline undulator for a fixed value of the undulator periods $N_{\mathrm{d}}=L_{\mathrm{d}}(C) / \lambda$ it is necessary to establish the ranges of $\varepsilon$, $a$ and $\lambda$ within which all the conditions, formulated in (11), are met. Once the ranges are found one can calculate the spectral-angular distribution of the energy emitted in the undulator. The results, presented below in this section, show, that for an electronbased crystalline undulator the energy of emitted photons is in the range $\hbar \omega \geq 10^{2}$ $\mathrm{keV}$. For such energies the values of the attenuation length for all crystals fall within the $\mathrm{cm}$ range [17], resulting in a strong inequality $L_{\mathrm{a}}(\omega) \gg L_{\mathrm{d}}(0)$. Therefore, it is the dechanneling which becomes the dominant effect restricting the length of the undulator (see the forth condition in (1)).

To perform the numerical analysis let us express $C, a$ and $\omega$ as functions of $\lambda$. From $N_{\mathrm{d}}=(1-C)^{2} L_{\mathrm{d}}(0) / \lambda$ one finds the following expression for the dependence $C(\lambda)$ :

$$
C(\lambda)=1-\sqrt{\frac{N_{\mathrm{d}} \lambda}{L_{\mathrm{d}}(0)}} .
$$

Because of the condition $C \geq 0$, the quantity $\lambda_{\max }=L_{\mathrm{d}}(0) / N_{\mathrm{d}}$ defines the maximum value of the undulator period.

Substituting $C=4 \pi^{2} \varepsilon a / U_{\max }^{\prime} \lambda^{2}$ into (7) one derives the dependence $a(\lambda)$ :

$$
a(\lambda)=\frac{\lambda^{2} U_{\max }^{\prime}}{4 \pi^{2} \varepsilon}\left(1-\sqrt{\frac{N_{\mathrm{d}} \lambda}{L_{\mathrm{d}}(0)}}\right) .
$$

From (7) and (8) follows that by tuning $\lambda$ and $\varepsilon$ for fixed $N_{\mathrm{d}} \gg 1$, one can establish the ranges of $a$ and $C$ where the first and second conditions in (1) are met for a given crystal.

Using the dependence $a(\lambda)$ it is possible to calculate the frequency of the fundamental harmonic as a function of $\lambda$ : $\omega_{1}(\lambda)=8 \pi \gamma^{2} c \lambda^{-1} /\left(2+p^{2}(\lambda)\right.$ ) (here $p(\lambda)=2 \pi \gamma a(\lambda) / \lambda$ is the undulator parameter). The spectral-angular distribution of undulator radiation in the forward direction at $\omega=\omega_{1}$ is calculated as follows $[9,12,18]$ :

$$
\left.\frac{\mathrm{d}^{3} E}{\hbar \mathrm{d} \omega \mathrm{d} \Omega}\right|_{\substack{\omega=\omega_{1} \\ \theta=0^{\circ}}}=4 \alpha \gamma^{2} N_{\mathrm{d}}^{2} z(1-2 z)\left[J_{0}(z)-J_{1}(z)\right]^{2},
$$

where $z=p^{2} / 2\left(2+p^{2}\right), J_{n}(z)$ is the Bessel function of integer order and $\theta$ is the emission angle with respect to the undulator axis.

Figures 4 and 5 present the results of numerical calculations of the dependences a, $C, \hbar \omega_{1}$ and $\mathrm{d}^{3} E / \gamma^{2} \mathrm{~d} \hbar \omega \mathrm{d} \Omega$ on $\lambda$ for $\varepsilon=50 \mathrm{GeV}$ electrons channeling in $\operatorname{Si}(111)$ and $\mathrm{C}(111)$. In figures 4 (a) and 5(a) the ratio $a / d$ versus $\lambda$ is shown for the fixed values of undulator periods within the dechanneling length. These graphs illustrate the ranges of $a, \lambda$ and $N_{\mathrm{d}}$ within which the second and third conditions from (1) are met. The curves 
presented in the figures suggest that the condition $a / d>1$ is fulfilled for $N_{\mathrm{d}} \leq 15$, i.e. the undulator with a sufficiently large number of periods can be considered.
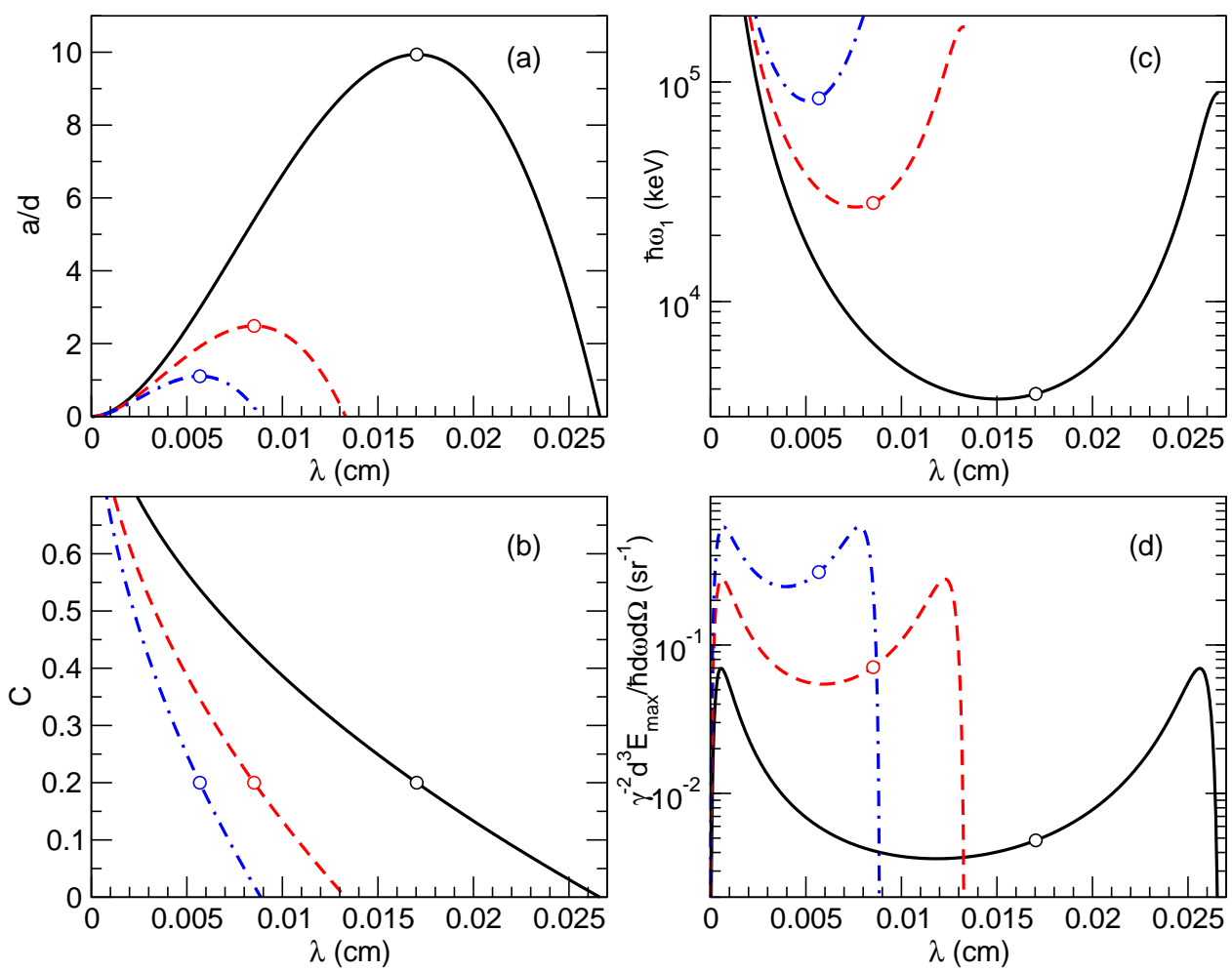

Figure 4. Dependences of $a / d, C, \hbar \omega_{1}$ and the peak intensity (9) on $\lambda$ for a 50 $\mathrm{GeV}$ electron channeling in a periodically bent $\operatorname{Si}(111)(d=2.35 \AA)$. In each graph the three curves correspond to different values of undulator periods within the dechanneling length: the solid curves stand for $N_{\mathrm{d}}=5$, the dashed curves - for $N_{\mathrm{d}}=10$, the chained curves - for $N_{\mathrm{d}}=15$. For each $N_{\mathrm{d}}$ the open circles indicate the parameters of undulator with $C=0.2$ (see graph (b)). This $C$ value ensures the maximum of the ratio $a / d$ (graph (a)).

Comparing figures 4(a) and 5(a) one notices that the curves for $\mathrm{C}(111)$ produce higher values of the ratio $a / d$ than those for $\mathrm{Si}(111)$ calculated for the same number of periods. Let us explain this difference. It follows from (8) that the ratio $a / d$ as a function of $\lambda$ attains maximum at $\lambda=16 \lambda_{\max } / 25$. The maximum value of the ratio is given by

$$
\left(\frac{a}{d}\right)_{\max }=\frac{4^{3}}{5^{5} \pi^{2} N_{\mathrm{d}}^{2} \varepsilon} \frac{U_{\max }^{\prime} L_{\mathrm{d}}^{2}(0)}{d} .
$$

For fixed values of $\varepsilon$ and $N_{\mathrm{d}}$ the magnitude of $(a / d)_{\max }$ depends on the parameters of a channel, $U_{\max }^{\prime}$ and $d$, and on the dechanneling length $L_{\mathrm{d}}(0)$, equation 2 . Taking into account that $U_{\max }^{\prime}$ and $d$ equal to $9.2 \mathrm{GeV} / \mathrm{cm}$ and $1.54 \AA$ for $\mathrm{C}(111)$ and to $8.0 \mathrm{GeV} / \mathrm{cm}$ and $2.35 \AA$ for $\operatorname{Si}(111)$ (see [19]), and $L_{\mathrm{d}}(0) \approx 0.16 \mathrm{~cm}$ for $\mathrm{C}$ and $L_{\mathrm{d}}(0) \approx 0.13 \mathrm{~cm}$ for $\mathrm{Si}$ (see figure 2), one finds that the ratio $(a / d)_{\max }$ for $\mathrm{C}(111)$ is approximately 2.4 times higher than that for $\operatorname{Si}(111)$. 

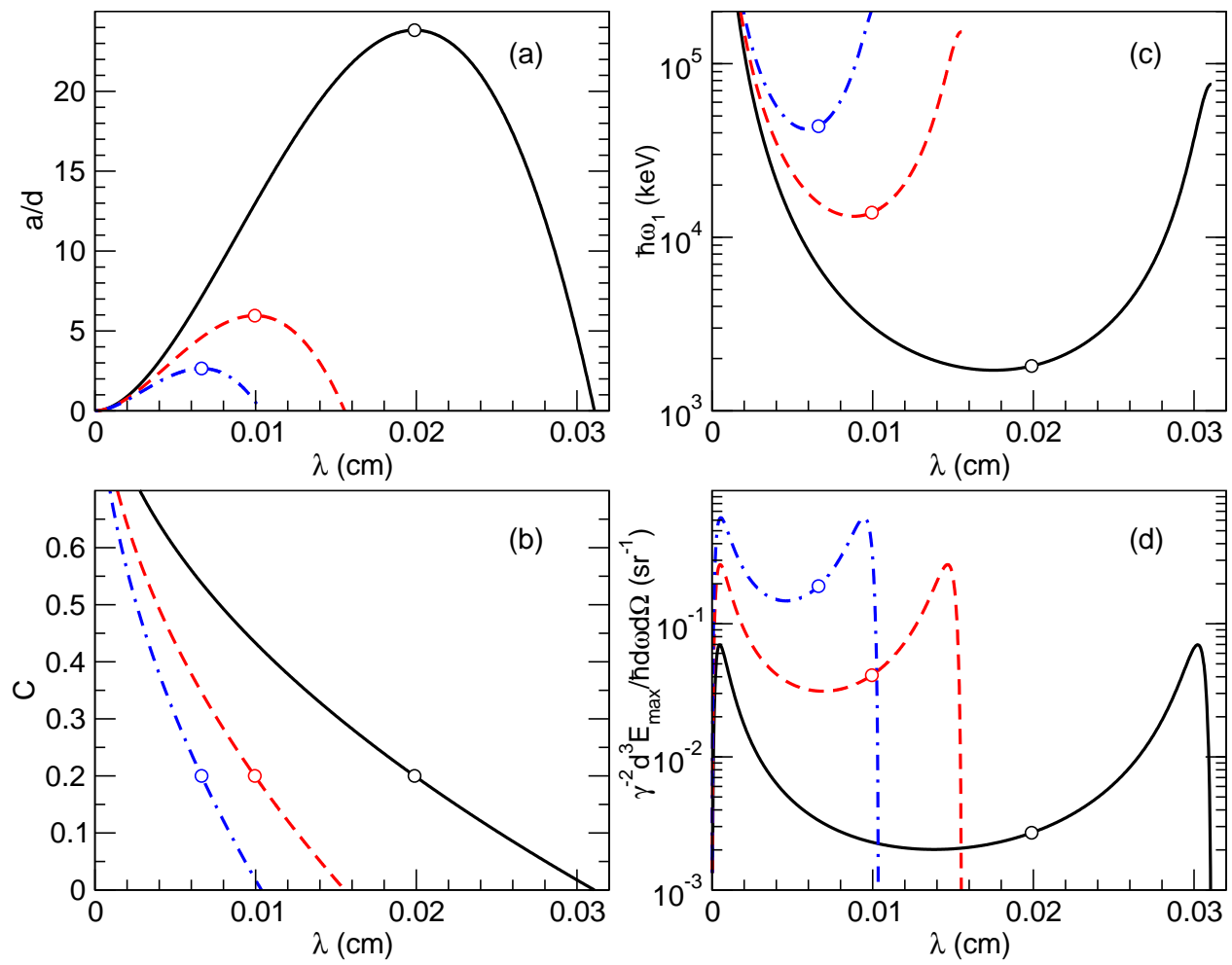

Figure 5. Same as in figure 4 but for $\mathrm{C}(111)$.

One can easily demonstrate that the ratio $(a / d)_{\max }$ is reached when $C=0.2$. This is valid for all $N_{\mathrm{d}}$ (see figures 4 and 5, where open circles mark the parameters corresponding to this value of $C$ ). The graphs (a) and (b) in figures 4 and 5 allow one to explicitly establish the ranges of parameters within which the conditions (1) are fulfilled, and consequently, the operation of an electron-based undulator is feasible. These ranges are: $C \leq 0.2, N_{\mathrm{d}} \simeq 10, \lambda \approx 10^{1} \ldots 10^{2} \mu \mathrm{m}$ and $a \approx 2 \ldots 20 \AA$. Let us note that the indicated ranges of $\lambda$ and $a$ are close to those which were established for a positron-based undulator (see, e.g., [3]).

Graphs (c,d) in figures 4 and 5 present the dependences on $\lambda$ of the energy fundamental harmonic (graph (c)) and of the peak intensity (9) calculated in the forward direction at $\omega=\omega_{1}$ and scaled by the factor $\gamma^{2}$, graph (d). These graphs demonstrate that within the $a, \lambda$ and $N_{\mathrm{d}}$ ranges indicated above, the magnitude of $\hbar \omega$ and of the intensity of undulator radiation can be varied by the orders of magnitude.

Figures 4(c) and 5(c) indicate that the energy of photons emitted in the $50 \mathrm{GeV}$ electron-based crystalline undulator lies within the $1 \ldots 10^{2} \mathrm{MeV}$ range. Let us note here, that the corresponding values of the attenuation lengths (for both $\mathrm{C}$ and $\mathrm{Si}$ crystals) exceed $5 \mathrm{~cm}$ (see, e.g. [16,17]). This value by far exceeds the dechanneling lengths in the crystals. Therefore, the statement on the negligible role of the photon attenuation, made in the opening paragraph of this section, is fully justified.

Let us now discuss the extent to which the decrease of the electron energy influences the allowed ranges of parameters of the crystalline undulator. Figure 6 presents the 
dependences of $a / d, C$, $\hbar \omega_{1}$ and the peak intensity on $\lambda$ for the electron of a lower energy, $\varepsilon=20 \mathrm{GeV}$, channeling in $\mathrm{C}(111)$.
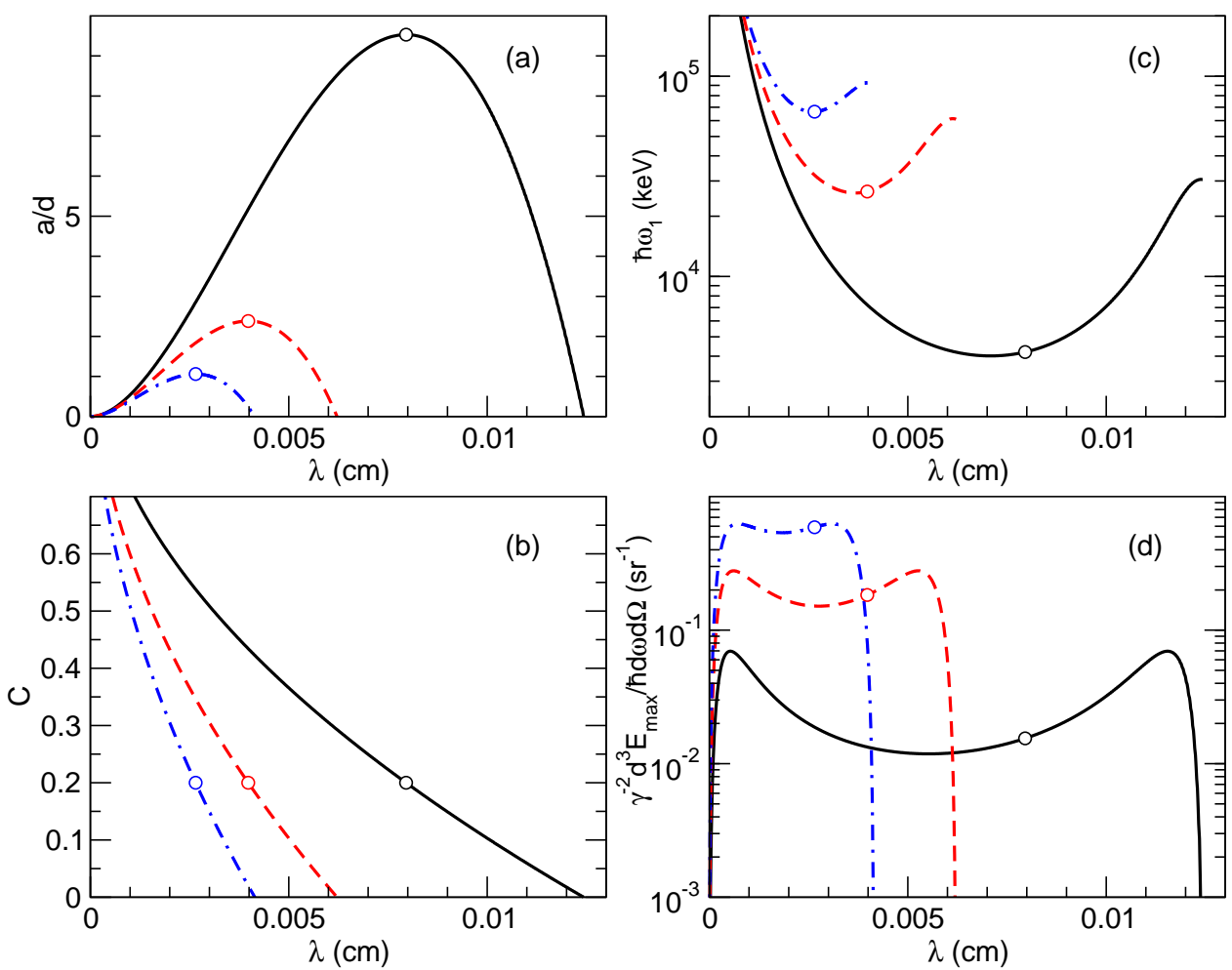

Figure 6. Same as in figure团but for a $20 \mathrm{GeV}$ electron channeling in the periodically bent C(111).

Comparing the curves in figures 6(a,b) with the corresponding dependences from figures 5 (a,b) one notices, that the domain of parameters $a / d, \lambda$ and $N_{\mathrm{d}}$ consistent with the conditions from (11) shrinks with the decrease of $\varepsilon$. Firstly, it is seen that the undulator period for a $20 \mathrm{GeV}$ electron is noticeable smaller than that for a $50 \mathrm{GeV}$ one. This feature is a corollary of a linear dependence of the dechanneling length on $\varepsilon$ (see (5)). As a result, the maximum value of the undulator period, $\lambda_{\max }=L_{\mathrm{d}}(0) / N_{\mathrm{d}}$, consistent with the condition $0 \leq C \leq 1$, is 2.5 times less for a $20 \mathrm{GeV}$ electron. Due to the same reason the maximal values of $a / d$ in figure 6(a) are 2.5 times lower than those in figure 5(a). Indeed from (5) and (10) follows, that for fixed $N_{\mathrm{d}}$ and for the same channel $(a / d)_{\max } \propto L_{\mathrm{d}}^{2}(0) / \varepsilon \propto \varepsilon$. As a result, in the case of $20 \mathrm{GeV}$ electrons the condition $a / d>1$ can be satisfied only within the reduced interval of the undulator periods, $N_{\mathrm{d}}<10$.

The arguments, presented above, indicate that further decrease of $\varepsilon$ will result, eventually, in a collapse of the domain of the parameters consistent with (11). To illustrate this, in figure 7 we present the dependence of the ratio $a / d$ on $\lambda$ for a 1 $\mathrm{GeV}$ electron. This figure illustrates that the case $N_{\mathrm{d}} \gg 1$ can be realized only if $a / d<1$, which contradicts to the second condition from (1). For such low electron energies the large-amplitude regime can be realized only for $N_{\mathrm{d}} \sim 1$. 


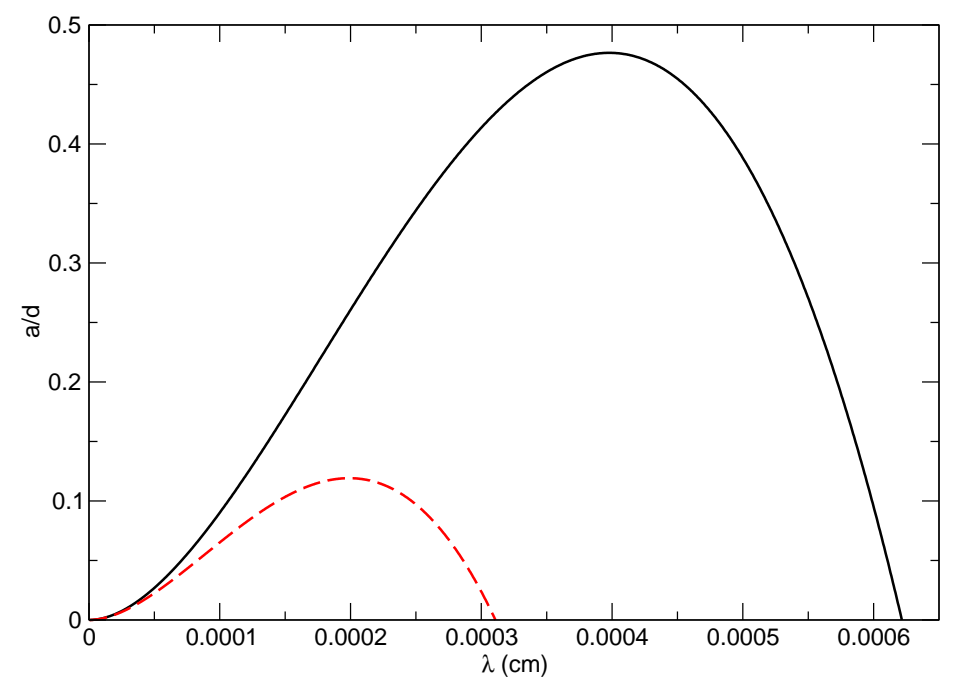

Figure 7. Dependence of $a / d$ on $\lambda$ for a $1 \mathrm{GeV}$ electron channeling in a periodically bent $\mathrm{C}(111)$. The curves correspond to different values of undulator periods within the dechanneling length: the solid curve stands for $N_{\mathrm{d}}=5$, the dashed curve - for $N_{\mathrm{d}}=10$.

Figures 446] allow one to define a set of parameters which characterize the undulator and its radiation. For example, fixing $N_{\mathrm{d}}$ and $C$ one finds: the period $\lambda$ - from graphs (b), the amplitude $a$ - from graphs (a), $\hbar \omega_{1}$ and the peak intensity - from graphs (c) and (d). In each figure, open circles mark the parameters of undulators with different number of $N_{\mathrm{d}}$ but with the same value of $C$ equal to 0.2 . For the undulators, based on the electron channeling in $\mathrm{C}(111)$, we calculated the spectral distribution of radiation (in the forward direction) in vicinity of the corresponding fundamental harmonics, i.e. for $\omega \sim \omega_{1}$. To calculate the distributions we followed the formalism, developed in [9], which describes the undulator radiation in presence of the dechanneling and the photon attenuation. Narrow peaks in figure 8 represent the results of these calculations. Wide peak in each graph stands for the spectral distribution of the channeling radiation in the forward direction. To obtain the latter we, at first, calculated the spectra for individual trajectories (using the Pöschl-Teller model [18] for the interplanar potential), corresponding to a stable channeling for given $C$. Then, the averaging procedure was carried out to calculate the spectra (see [5,6] for the details). Figure 8 clearly demonstrates that by tuning the parameters of bending and varying the electron energy it is possible to separate the frequencies of the undulator radiation from those of the channeling radiation, and to make the intensity of the former comparable or higher than of the latter.

\section{Conclusion}

We have demonstrated that it is feasible to devise an undulator based on the channeling effect of ultra-relativistic electrons in a periodically bent crystal. The electron-based 

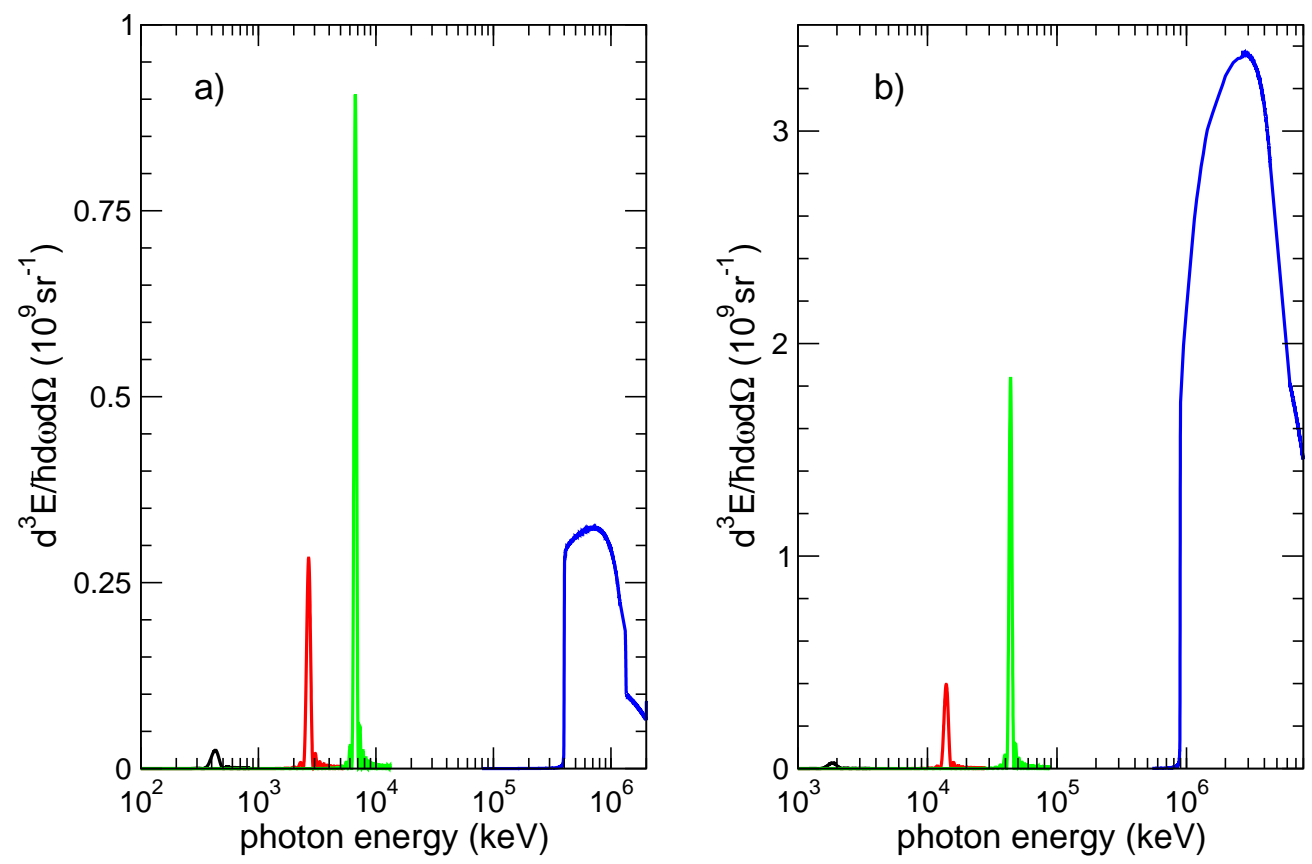

Figure 8. Spectral distributions of the undulator and channeling radiation emitted in the forward direction by a $20 \mathrm{GeV}$ (figure (a)) and by a $50 \mathrm{GeV}$ (figure (b)) electron in $\mathrm{C}(111)$. Narrow peaks stand for the spectral distribution of the undulator radiation in the vicinity of the fundamental harmonics for six different undulators (corresponding to $C=0.2$ ) defined by open circles in figures 5 and 6 . In each graph the first (i.e., the leftest) narrow peak corresponds to $N_{\mathrm{d}}=5$, the second peak - to $N_{\mathrm{d}}=10$, and the third peak - to $N_{\mathrm{d}}=15$.

undulator operates in the tens of $\mathrm{GeV}$ range of electron energies. These energies are noticeably higher than those in a positron-based undulator. Apart from the difference in energies of the projectiles, other parameters of the crystalline undulators (i.e., $a, p, \lambda$ ) are much alike. Therefore, to construct an electron-based undulator one can consider the methods proposed earlier in connection with a positron-based undulator. These methods include propagation of an acoustic wave $[2,3,28]$ or the use of a graded composition of different layers [29] or a periodic mechanical deformation of the crystalline structure [21].

Present technologies allow one to construct the periodically bent crystalline structures with the required parameters [10]. Similar to the case of a positron-based undulator $[9,10]$, the parameters of high-energy electrons beams available at present (see Ch. 26 in [16]) are sufficient to achieve the necessary conditions to construct the undulator and to create, on its basis, powerful radiation sources in the $\gamma$-region of the spectrum. As in the positron case $[2,3,8]$ it is meaningful to explore the idea of a $\gamma$-laser by means of an electron-based undulator.

This work has been supported by the European Commission (The PECU project, contract No. 4916). 


\section{References}

[1] Tabrizi M, Korol A V, Solov'yov A V and Greiner W 2006 Submitted to Phys. Rev. Lett (Preprint: physics/0611012)

[2] Korol A V, Solov'yov A V and Greiner W 1998 J. Phys. G: Nucl. Part. Phys. 24 L45

[3] Korol A V, Solov'yov A V and Greiner W 1999 Int. J. Mod. Phys. E 849

[4] Kumakhov M A and Komarov F F 1989 Radiation From Charged Particles in Solids (New York: AIP)

[5] Krause W, Korol A V , Solov'yov A V and Greiner W 2000 J. Phys. G: Nucl. Part. Phys. 26 L87

[6] Korol A V, Solov'yov A V and Greiner W 2000 Int. J. Mod. Phys. E 977

[7] Korol A V, Solov'yov A V and Greiner W 2001 J. Phys. G: Nucl. Part. Phys. 2795

[8] Korol A V, Solov'yov A V and Greiner W 2004 Int. J. Mod. Phys. E 13867

[9] Korol A V, Solov'yov A V and Greiner W 2005 Proc. SPIE - Int. Soc. Opt. Eng. 5974597405

[10] http://ec.europa.eu/research/fp6/nest/pdf/nest_projects_september_2005_preview.pdf (pp. 31-32)

[11] Lindhard J 1965 Kong. Danske Vid. Selsk. Mat.-Phys. Medd. 3414

[12] Alferov D F, Bashmakov Yu A and Cherenkov P A 1989 Usp. Fiz. Nauk 157389 (Engl. Trans. 1989 Sov. Phys.-Uspekhi 32 200)

[13] Barbini R et al 1990 Riv. Nuov. Cim. 131

[14] Rullhusen P, Artru X and Dhez P 1998 Novel Radiation Sources Using Relativistic Electrons (Singapore: World Scientific)

[15] Kumakhov M A 1986 Radiation of the Channeled Particles in Crystals (Moscow: Energoatomizdat)(in Russian)

[16] Yao W M et al 2006 J. Phys. G 331

[17] Hubbel J H and Seltzer S M Table of X-Ray Mass Attenuation Coefficient NISTIR 5632 - Web Version http://physics.nist.gov/xaamdi

[18] Baier V N, Katkov V M and Strakhovenko V M 1998 High Energy Electromagnetic Processes in Oriented Single Crystals (Singapore: World Scientific)

[19] Biruykov V M, Chesnokov Y A and Kotov V I 1996 Crystal Channeling and its Application at High Energy Accelerator (Berlin: Springer)

[20] Uggerhøi U I 1980 Rev. Mod. Phys. 771131

[21] Bellucci et al 2003 Phys. Rev. Lett. 90034801

[22] Baranov V T et al 2005 Pis'ma Zh. Eks. Teor. Fiz. 82638 (Engl. Tran. Sov. JETP Lett. 82 562)

[23] Baier V N, Katkov V M and Fadin V.S. 1973 Radiation by Relativistic Electrons (Moscow: Atomizdat)(in Russian)

[24] Carrigan R A, Jr. 2006 Fundamental channeling questions at ultra relativistic energies FERMILABCONF-06-310-AD

[25] Adejshvili D I et al 1984 Pis'ma Zh. Tekh. Fiz. 10197 (Engl. Tran. Sov. Phys. - Tech. Phys. Lett. $1082)$

[26] Adejshvili D I et al 1985 Radd. Eff. Lett. 87135

[27] Komaki K et al 1984 Nucl. Instr. Meth. B 271

[28] Kaplin V V, Plotnikov S V and Vorobiev S A 1980 Sov. Phys. - Tech. Phys 25650

[29] Mikkelsen U and Uggerhøi 2000 Nucl. Inst. Meth. B 160435 